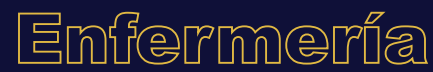

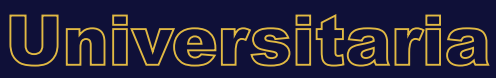

\section{Fomento del autocuidado al paciente con sondaje vesical domiciliario mediante sesiones educativas}

Promotion of self-care in home patients with urinary catheter through educational interventions

\section{Fomento do autocuidado para o paciente com sonda vesical domiciliar mediante sessões educativas}

\section{E. Moreno-Cabelloa,*, A.M. Gónzalez-Picazo ${ }^{\mathrm{b}}$, C. Martín-Salinasc}

ORCID

a $0000-0002-6480-6463$

b $0000-0002-4038-8260$

c $\underline{0000-0002-2702-8797}$

Universidad Autónoma de Madrid, Facultad de Medicina, Departamento de Enfermería, Madrid, España

Recibido: 04 septiembre 2019

Aceptado: 16 enero 2020

RESUMEN

Introducción: La infección tardía es una posible complicación del implante de un esfínter urinario artificial (método más aceptado para tratar la incontinencia urinaria de esfuerzo secundaria a prostatectomía radical). Si sucede, debe retirarse la prótesis y colocar una sonda vesical hasta su reinserción meses más tarde. El paciente se convierte en portador

*Autora para correspondencia. Correo electrónico: esther.morenocabello@estudiante.uam.es https://doi.org/10.22201/eneo.23958421e.2020.2.736

1665-7063/@ 2020 Universidad Nacional Autónoma de México, Escuela Nacional de Enfermería y Obstetricia. Este es un artículo Open Access bajo la licencia CC BY-NC-ND (http://creativecommons.org/licenses/by-nc-nd/4.o/). 
domiciliario de sonda vesical, que puede adquirir una infección de tracto urinario por un mal cuidado.

Objetivo: Desarrollar sesiones educativas para que un paciente adquiera destreza en el manejo de sonda vesical y consecuentemente, prevenir las infecciones asociadas.

Desarrollo: Se presenta el caso de un varón de 80 años, portador de sonda vesical por retirada temporal de su esfínter urinario artificial. Al realizar la valoración de enfermería, se identifica el diagnóstico enfermero Conocimientos deficientes en relación con los cuidados del dispositivo. Posteriormente, se seleccionan los resultados esperados y se planifican las intervenciones educativas. Tras llevarlas a cabo identificamos que el paciente incrementó sus conocimientos sobre el manejo de la sonda vesical.

Conclusiones: La educación sanitaria es una herramienta de prevención del riesgo de infección asociado al mantenimiento de la sonda vesical en el domicilio, ya que, si el paciente no es capaz de afrontar su autocuidado, el tratamiento pierde efectividad. Es imprescindible identificar las necesidades formativas de los pacientes, abordarlas mediante intervenciones educativas reforzadas con documentación escrita durante el ingreso, para proporcionar la información necesaria y fortalecer su comprensión, por lo que puede mejorar el nivel de autocuidado, así como prevenir complicaciones tras el alta hospitalaria.

Palabras clave: Educación sanitaria; alta del paciente; catéter urinario; autocuidado; España.

\section{ABSTRACT}

Introduction: Late infection is a possible complication an artificial urinary sphincter's implant the most accepted method to treat urinary incontinence secondary to radical prostatectomy. If this happens, the prosthesis must be removed, and a urinary catheter was placed. Then, the patient becomes a urinary catheter home carrier prone to acquire urinary tract infections as a result of carelessness.

Objective: To develop educational interventions so that these patients acquire dexterity to manage the urinary catheter and thus, prevent the possible associated infections.

Development: A case of an 80 years old male carrying a urinary catheter temporarily substituting an artificial urinary sphincter is addressed. The nursing diagnosis assessment is performed. The patient demonstrates Insufficient knowledge related to the management of the device. Educational interventions addressing the situation are planned. After the implementation of the strategy, the patient improves his knowledge of urinary catheter management.

Conclusions: Health education is an infection risk prevention tool that can address the management of urinary catheters in-home patients, improving the effectiveness of the related treatments. It is essential to identify the needs of the patients and help address them through educational interventions to enhance self-care and prevent possible complications. Keywords: Health education; hospital discharge; urinary catheter; self-care; Spain.

\section{RESUMO}

Introdução: A infeção tardia é uma possivel complicação do implante de um esfíncter urinário artificial (método mais aceito para tratar a incontinência urinaria de esforço 
secundária a prostatectomia radical). Se acontecer, deve retirar-se a prótese e colocar uma sonda vesical até sua reinserção meses mais tarde. O paciente torna-se portador domiciliar de sonda vesical, podendo adquirir uma infeção de tracto urinário por um mal cuidado.

Objetivo: Desenvolver sessões educativas para que um paciente adquira destreza no manejo de sonda vesical e consequentemente, prevenir as infecções associadas.

Desenvolvimento: Presenta-se o caso de um homem de 80 anos, portador de sonda vesical por retirada temporal de seu esfíncter urinário artificial. Realiza-se a valoração de enfermagem, onde se identifica o diagnóstico de enfermagem Conhecimentos deficientes ao respeito dos cuidados do dispositivo. Posteriormente, selecionam-se os resultados esperados e planificam-se as intervenções educativas. Após levá-las a cabo, consegue-se que o paciente incremente seus conhecimentos sobre o manejo da sonda vesical.

Conclusões: A educação sanitária é uma ferramenta de prevenção do risco de infeção associado à manutenção da sonda vesical na residência, já que, se o paciente não for capaz de afrontar seu autocuidado, o tratamento perde efetividade. É imprescindível identificar as necessidades formativas dos pacientes e combatê-las mediante intervenções educativas reforçadas com documentação escrita durante o ingresso, para proporcionar a informação necessária e reforçar sua compreensão, pelo que pode melhorar o nível de autocuidado e prevenir complicações após a alta hospitalar.

Palavras-chave: Educação sanitária; alta do paciente; catéter urinário; autocuidado; Espanha.

\section{INTRODUCCIÓN}

La Incontinencia Urinaria de Esfuerzo (IUE) es una secuela importante del tratamiento quirúrgico del cáncer de próstata ${ }^{1}$. La International Continence Society (ICS)², la define como cualquier pérdida involuntaria de orina. Se considera que puede afectar en mayor o menor medida la calidad de vida de cualquier individuo, con gran impacto social, psicológico, laboral, sexual, y económico3.

La causa más frecuente de incontinencia urinaria masculina es la lesión del sistema esfinteriano proximal, generalmente después de una prostactectomía radical ${ }^{4}$. El porcentaje de afectados varía según el grado de pérdida de orina que se considere como problemático, el $5 \%$ en los pacientes necesitan uso de pañal y asciende hasta el $49 \%$ si se trata de pérdidas de apenas unas gotas. Sin embargo, si hablamos de IUE, se presenta en un 5-25\% de los pacientes sometidos a este tratamiento. Cabe señalar que este rango tan amplio en la incidencia se debe a los diferentes métodos utilizados para cuantificarla 5 .

La importancia o gravedad de la incontinencia post-prostatectomía radical se determina por su impacto sobre la calidad de vida del paciente y las posibilidades terapéuticas que se deben adaptar al individuo en relación con la causa, grado, efecto sobre la calidad de vida, así como expectativas frente al tratamiento. No obstante, el Esfínter Urinario Artificial (EUA) es actualmente el tratamiento de primera elección para abordar la incontinencia urinaria post-prostatectomía radical con tasas de éxito superiores al 80\% ${ }^{6}$. Diseñado por Scott et al..$^{7}$ en 1973 , ha experimentado numerosas modificaciones, pero mantiene el principio básico que consiste en un sistema hidráulico con un reservorio o balón regulador de la presión del sistema, un manguito que rodea la uretra y una bomba de activación que se coloca en el escroto. El sistema se encuentra lleno por un líquido isosmótico, que es el facilitador del funcionamiento de esta prótesis. Si no se activa la bomba, el líquido 
se mantiene en el manguito, ocluyendo la uretra e impidiendo de este modo el paso de la orina. Cuando la vejiga se llena y la persona acciona la bomba de control, el líquido cambia de sentido, pasa del manguito al reservorio, desinflándose el primero y descomprimiéndose la uretra.

A pesar de que el esfínter urinario artificial AMS-80o ha sido durante las dos últimas décadas una adecuada opción terapéutica para el tratamiento de la incontinencia urinaria de esfuerzo refractaria a otros tratamientos, la implantación de la prótesis no esta exenta de complicaciones. La infección tardía, causada generalmente por Staphylococcus epidermidis, provoca la retirada de la prótesis y la utilización de una Sonda Vesical (SV) hasta la resolución del proceso infeccioso para luego colocar un nuevo esfínter artificial, entre 3 y 6 meses después 5 .

Por otro lado, la infección del tracto urinario es la complicación más frecuente asociada a la SV y se relaciona, entre otros factores, con el tiempo de permanencia de la sonda ${ }^{8}$. El riesgo de desarrollar una Infección del Tracto Urinario (ITU) se muestra solamente al insertar la SV y se presenta en el 10-20\% de los pacientes sometidos a un cateterismo permanente ${ }^{9-10}$. Se estima que más del $80 \%$ de las ITU están relacionadas con la SV, aunque se desconoce la incidencia en pacientes ambulatorios ${ }^{11}$ que también tienen riesgo de adquirir una infección, debido a que el domicilio es un ambiente no controlado. A pesar de la falta de estudios concluyentes, algunos autores informan que más del 3\% de los pacientes con SV domiciliaria contrae una infección ${ }^{12}$.

En este sentido, los pacientes sometidos a una prostactectomía radical son dados de alta del hospital entre 1-2 días después de la operación y son los encargados de realizar los autocuidados de la SV, manifestando una elevada ansiedad e incertidumbre por el escaso conocimiento y manejo que tienen de este dispositivo ${ }^{13}$. Por ello previo al alta hospitalaria, es necesaria la formación del paciente, así como de la familia en la cual se proporcione información y recomendaciones, tanto de forma oral como escrita, destacando con un lenguaje claro, sencillo, sin tecnicismos la importancia de la higiene personal; de las medidas de asepsia, de la manipulación de la sonda que puede influir positivamente en los conocimientos del paciente para su autocuidado y contribuir a disminuir el riesgo de infección 9,14 .

La Educación para la Salud (EpS), es un proceso formativo mediante el cual individuos y grupos de personas pueden adoptar conductas y estilos de vida que favorece la promoción, el mantenimiento o la restauración de la salud ${ }^{15}$, lo que les convierte en agentes activos de su propia salud. Requiere una combinación de técnicas de enseñanza que incluya una etapa tanto de diseño como de planificación, una de intervención y una etapa final de evaluación de la intervención, además de estar en correspondencia con las necesidades educativas de los receptores ${ }^{16}$.

Aunado a la colocación de una SV y el periodo de espera en el domicilio hasta la colocación de un nuevo esfínter artificial, se desarrolla este caso clínico derivado de las nuevas demandas de autocuidado por parte del paciente. Dicho caso consistió en un programa educativo orientado a proporcionar a este los conocimientos y habilidades necesarias para que pueda gestionar su nueva situación de salud ${ }^{17}$. Como resultado, el objetivo del presente caso clínico fue desarrollar sesiones de educación para la salud dirigidas a promover el autocuidado y evitar infecciones en un paciente con sonda vesical en su domicilio.

\section{DESARROLLO}

\section{Presentación del caso}

Varón de 80 años que ingresa al servicio de Urología por infección de su esfínter urinario artificial. En el momento del ingreso el paciente tiene fiebre $\left(38.2^{\circ} \mathrm{C}\right.$ ), dolor en reposo (puntuando 
5 en la escala visual analógica $\left.[\mathrm{EVA}]^{18}\right)$ y una inflamación muy notoria en la zona de la bomba, acompañada de enrojecimiento. Es portador de una talla vesical a bolsa, con orina clara. Como consecuencia de la infección secundaria a la prótesis, se retira en el quirófano; el paciente espera con una SV en el domicilio, entre tres y seis meses para colocar un nuevo EUA.

\section{Plan de cuidados enfermero}

El proceso de cuidado enfermero aplica el modelo científico a la práctica asistencial para proporcionar cuidados de forma racional, sistemática y basada en la evidencia científica. Inicia con la valoración de la situación de salud mediante la teoría de Dorothea Orem ${ }^{19}$ que proporciona información para la identificación de diagnósticos enfermeros ${ }^{20}$, la selección de criterios de resultados (NOC $)^{21}$ y de intervenciones (NIC)22; finaliza con la planificación de actividades individualizadas y la evaluación de todo el proceso.

\section{Valoración según la teoría de Dorothea Orem}

Los datos registrados a continuación se obtuvieron el mismo día de la intervención quirúrgica, a su regreso a la unidad de urología después de cuatro horas en la unidad de recuperación postanestésica,y tras obtener su consentimiento para realizar la entrevista.

- Factores condicionantes básicos. Varón con dos hijos casados y tres nietos adolescentes. Desde que quedó viudo hace 12 años, ha preferido vivir solo en su casa, aprendió a organizar las tareas domésticas. Sus hijos y nietos le visitan regularmente pero no quiere trasladarse a vivir con ninguno de ellos. Se acuesta tarde viendo la televisión; duerme bien, entre 7 y 8 horas.

Tiene varios amigos viudos como él que se reúnen en el centro social de su barrio, prácticamente todas las mañanas.

- Requisitos de autocuidado: Estado general de salud. Prostatectomía radical en 2008 por cáncer de próstata, en remisión completa desde 2010, presentando IUE desde 2008 s/a prostatectomía radical. Se le colocó un esfínter urinario artificial AMS 800 en 2012. Hernia hiatal + Enfermedad por reflujo gastroesofágico + Esófago de Barrett (sin fecha conocida de inicio). Sin antecedentes de reacciones adversas medicamentosas.

Una vez controlada la infección, motivo del ingreso, es intervenido quirúrgicamente para retirarle el EUA. El paciente se muestra tranquilo. Sus constantes están dentro del rango normal: FC 72 lpm, PA 125/78 mmHg, FR 18 rpm, SpO2 98\% basal y Ta $36.1^{\circ} \mathrm{C}$. Refiere dolor en la incisión quirúrgica de 6 EVA $^{18}$. Pesa $78.150 \mathrm{~kg}$, mide 1.69m, (índice de masa corporal: $27.4 \mathrm{Kg}$ / $\mathrm{m} 2$ ). Vía venosa periférica en miembro superior izquierdo sin signos de flebitis.

La talla vesical se ha retirado, ahora porta una SV para proteger las zonas que han sido manipuladas quirúrgicamente con orina ligeramente hematúrica. Deberá llevarla entre $15 \mathrm{y}$ 30 días si no existe complicaciones. Al principio, el alta esta prevista a las 48 horas de la intervención.

- Requisitos de autocuidado: Desarrollo. El paciente esta adaptado a su etapa vital, no observándose déficit en este aspecto.

- Requisitos de autocuidado: Universales. Clasificados en la tabla 1. La Agencia de Enfermería muestra la necesidad de suplencia total en los requisitos de alimentación (fluidoterapia y pendiente de iniciar tolerancia), además de prevención de peligros (vía venosa periférica y sonda vesical); suplencia parcial en los requisitos de eliminación intestinal (reposo en cama, proporcionar cuña); higiene, vestido y arreglo personal (reposo 
en cama, proporcionar útiles de aseo); así como, movilización y mantenimiento de una buena postura corporal (cambios de posición y recordarle los ejercicios de los miembros inferiores).

\section{Diagnósticos Enfermeros (DE)}

- Conocimientos deficientes relacionado con $(\mathrm{r} / \mathrm{c}$ ) desconocimiento de los cuidados de la sonda vesical manifestado por $(\mathrm{m} / \mathrm{p})$ demanda de información

- Riesgo de úlcera por presión r/c disminución de la movilidad y presión sobre prominencia ósea

- Déficit de autocuidado: Aseo, vestido r/c reposo en cama

- Problema de colaboración (PC): Infección

- PC: Dolor abdominal.

Aunque se han identificado varios problemas en este paciente, se optó por el DE Conocimientos deficientes ya que el paciente se irá de alta a su domicilio con la sonda vesical, vive solo y desconoce las precauciones que debe adoptar para un mantenimiento seguro de la sonda. Por lo tanto, es fundamental proporcionar una formación previa para evitar la aparición de complicaciones secundarias a un uso incorrecto, como podría ser una posible infección.

\section{Tabla 1. Valoración de la Agencia de Autocuidado Universal}

\begin{tabular}{|c|c|}
\hline $\begin{array}{l}\text { Mantenimiento de un ingreso } \\
\text { suficiente de aire }\end{array}$ & $\begin{array}{l}\text { Vía aérea permeable. Respiración rítmica abdominal. No disnea, tos ni } \\
\text { expectoración. } \\
\text { Buena coloración de piel y mucosas. }\end{array}$ \\
\hline $\begin{array}{l}\text { Mantenimiento de una ingesta } \\
\text { suficiente de líquidos }\end{array}$ & $\begin{array}{l}\text { Bien hidratado. Fluidoterapia intravenosa con solución salina fisiológica } 0,9 \% \text { a } \\
84 \mathrm{ml} / \mathrm{h} \text {. } \\
\text { Ausencia de edemas. }\end{array}$ \\
\hline $\begin{array}{l}\text { Mantenimiento de una ingesta } \\
\text { suficiente de alimentos }\end{array}$ & $\begin{array}{l}\text { Dieta absoluta. Pendiente de iniciar tolerancia oral con líquidos. } \\
\text { Tiene prótesis dental, en buenas condiciones. } \\
\text { Cribado nutricional sin existencia de riesgo. } \\
\text { Precisa asistencia en la alimentación. }\end{array}$ \\
\hline $\begin{array}{l}\text { Provisión de cuidados } \\
\text { asociados con los procesos de } \\
\text { eliminación }\end{array}$ & $\begin{array}{l}\text { Eliminación urinaria. SV Foley ( } \mathrm{n} \text { - 20), ligera hematuria. Diuresis } 200 \mathrm{ml} \text {. No } \\
\text { sudoración. } \\
\text { Eliminación intestinal. No ha hecho deposición después de la intervención } \\
\text { quirúrgica. }\end{array}$ \\
\hline $\begin{array}{l}\text { Mantenimiento del equilibrio } \\
\text { entre la actividad y el reposo }\end{array}$ & $\begin{array}{l}\text { Reposo en cama durante } 24 \text { horas. } \\
\text { Duerme unas } 7-8 \text { horas. No esta cansado ni somnoliento. }\end{array}$ \\
\hline $\begin{array}{l}\text { Mantenimiento del equilibrio } \\
\text { entre soledad y la interacción } \\
\text { social }\end{array}$ & $\begin{array}{l}\text { Llega de la URPA* acompañado por sus hijos y una nuera. } \\
\text { Esta orientado en las tres esferas, se muestra tranquilo y cómodo durante la } \\
\text { entrevista. Muy expresivo, abierto y con respuestas firmes. }\end{array}$ \\
\hline $\begin{array}{l}\text { Prevención de peligros para } \\
\text { la vida, el funcionamiento y el } \\
\text { bienestar humano }\end{array}$ & $\begin{array}{l}\text { Consciente y orientado en las tres esferas. } \\
\text { Tiene tres incisiones, una a nivel vesical, otra en la zona púbica y la tercera en la } \\
\text { zona perineal, con buen aspecto. } \\
\text { Dolor abdominal (EVA } 6) \text {. } \\
\text { Norton: } 15^{23} \text {. }\end{array}$ \\
\hline $\begin{array}{l}\text { Promoción del funcionamiento y } \\
\text { desarrollo humano }\end{array}$ & $\begin{array}{l}\text { Autoconcepto: Alegre y optimista. Afrontamiento positivo de la enfermedad. } \\
\text { Desconoce el funcionamiento, complicaciones, medidas de prevención y signos } \\
\text { de alarma relacionados con el manejo de la SV. Demanda información. } \\
\text { Se siente decepcionado con explicaciones previas (vagas, rápidas, sin comprobar } \\
\text { los conocimientos, entrega de folletos). } \\
\text { Conoce el motivo por el que lleva la sonda y el riesgo de infección. }\end{array}$ \\
\hline
\end{tabular}

*Unidad de Recuperación Postanestésica 


\section{PLANIFICACIÓN}

Tras la identificación del diagnóstico enfermero Conocimientos deficientes (NANDA), se planifican los cuidados de enfermería considerados necesarios (Tabla 2).

\section{Tabla 2. NANDA: Conocimientos deficientes}

\begin{tabular}{|c|c|c|c|c|}
\hline Características y factores & $\begin{array}{l}\text { Alta al domicilio con sonda vesical } \\
\text { Desconoce los cuidados necesarios } \\
\text { Vive solo }\end{array}$ & & & \\
\hline Resultados NOC & Indicadores & P. basal & P. diana & P. final \\
\hline \multirow{3}{*}{$\begin{array}{l}\text { Conocimiento: Procedimiento } \\
\text { terapéutico }\end{array}$} & Uso correcto de la sonda & 1 & 5 & 5 \\
\hline & Cuidados de la sonda & \multirow{2}{*}{1} & \multirow{2}{*}{5} & \multirow{2}{*}{5} \\
\hline & Posibles efectos indeseables & & & \\
\hline Resultados NOC & Indicadores & P. basal & P. diana & P. final \\
\hline \multirow{3}{*}{$\begin{array}{l}\text { Conocimiento: Control de la } \\
\text { infección }\end{array}$} & Factores de riesgo & 1 & 5 & 5 \\
\hline & Prácticas para evitar la infección & 1 & 5 & 5 \\
\hline & Cuando consultar & 1 & 5 & 5 \\
\hline Resultados NOC & Indicadores & P. basal & P. diana & P. final \\
\hline \multirow{3}{*}{$\begin{array}{l}\text { Satisfacción del paciente con la } \\
\text { enseñanza }\end{array}$} & Características del autocuidado & 1 & 5 & 5 \\
\hline & Signos de complicaciones & 2 & 5 & 5 \\
\hline & Tiempo para el aprendizaje & 1 & 5 & 5 \\
\hline & INTERVENCIONES (NIC) & & & \\
\hline
\end{tabular}

Enseñanza individual. Actividades.

- Valorar el nivel actual de conocimiento del paciente

- Seleccionar los materiales educativos. En este caso se utilizará una sonda vesical como la que tiene colocada para mostrarle sus componentes y el funcionamiento

- Determinar los factores que afectan a la ingesta nutricional

- Explicar las características de la sonda vesical, los riesgos que conlleva y sus cuidados ${ }^{24}$ :

- Ducha diaria, higiene genital mínimo 2 veces/día, retirando el prepucio y regresándolo después de aclarar a su posición natural

- Higiene de manos antes de manipulación de sonda

- Beber abundantes líquidos (1.5-2l/día)

- Desconectar la bolsa solo cuando sea necesario. Vaciado c/8h, usando el grifo

- Sustituir la bolsa c/8 días o antes si rotura, hedor o excesivo sedimento

- Situar la bolsa por debajo de la vejiga (evitar reflujo)

- Fijar sonda para prevenir tirones

- Dar tiempo para que haga preguntas y exprese sus inquietudes

- Proporcionar folletos sobre recomendaciones al paciente con sondaje vesical

Enseñanza individual. Actividades ${ }^{25}$.

- ¿QUÉ PUEDE PASAR?:

- Espasmos vesicales y salida de orina alrededor de la sonda $\rightarrow$ NORMAL

- ITU r/c inserción, cuidado, mantenimiento, tiempo de permanencia

- SIGNOS DE CONSULTA en Atención Primaria:

- Picor, dolor o ardor

- Cambios en el aspecto de la orina o en su excreción

- SIGNOS DE ALARMA (debe acudir a Urgencias):

- Retención urinaria (imposibilidad de orinar)

- Fiebre > 38ㄷ

- Malestar general 


\section{EJECUCIÓN}

Desde el día de la intervención, hasta el alta hospitalaria y los siguientes dos días, se desarrollan las sesiones educativas, tanto en el turno de la mañana como en el de la tarde, con el fin de incrementar su nivel de conocimientos sobre el manejo de la SV para potenciar su autonomía de cara al alta hospitalaria. El paciente se muestra proactivo y abierto al aprendizaje durante cada sesión formativa, solventando de forma gradual los problemas con los que contaba respecto al manejo de dicha sonda.

\section{EVALUACIÓN}

Coincidimos con el planteamiento de De Arco-Canoles ${ }^{26}$ sobre el perfil profesional enfermero, capacitado para trabajar en el ámbito general del ejercicio de la enfermería, incluyendo la promoción de la salud, la prevención de la enfermedad y los cuidados integrales a las personas enfermas. En este sentido, la educación propuesta en dicho caso es un elemento central del cuidado ${ }^{27}$ que requiere disponer de los conocimientos y la sensibilidad respecto a lo que es importante, así como estar a la vanguardia para dar respuesta a las necesidades identificadas ${ }^{28}$.

De conformidad con Marcos Espino ${ }^{19}$, la valoración enfermera mediantela Teoría del Autocuidado, permite al personal de enfermería disponer de una visión integral de las demandas y necesidades de cuidado del paciente. La Atención de Enfermería fundamentada en el Proceso de Atención en Cuidados garantiza la calidad de estos y contribuye a reducir la probabilidad de que aparezcan complicaciones.

Así mismo, concordamos con Abasolo ${ }^{11}$ en que las infecciones del tracto urinario en pacientes portadores de SV constituyen un grave problema de salud, por el cual puede prevenirse mediante la educación de los enfermos como familiares antes del alta al domicilio con respecto a las medidas a llevar a cabo para un correcto mantenimiento de dicha sonda. Se ha demostrado que proporcionar al paciente información escrita refuerza el programa educativo realizado, mejora la comprensión de los cuidados y contribuye a facilitar en este la información necesaria para la potenciación de su autocuidado en el domicilio tras el alta hospitalaria. De igual forma, Islas-Salinas ${ }^{30}$ indica que un educador para la salud debe evaluar la capacidad del paciente, como se refleja en nuestra valoración, a fin de participar en su autocuidado y proporcionarle las herramientas necesarias para que obtenga conocimientos que le permitan gestionar su nueva situación de salud.

La realización de este plan de cuidado, a partir de la metodología enfermera y con lenguaje disciplinar, permite al paciente abordar una nueva situación de salud derivada del correcto mantenimiento de la sonda vesical y poder asumir con éxito su autocuidado. Tras el proceso formativo y en vísperas del alta, el paciente explica los cuidados, verbaliza las posibles consecuencias, los signos de alarma, así como comprender el funcionamiento de la SV. Además, muestra un alto grado de satisfacción con los conocimientos proporcionados, el método propedéutico y el tiempo utilizado para asegurar la comprensión del procedimiento. En este sentido, se ha obtenido una puntuación del cambio de +4 en todos los criterios de resultado utilizados para evaluar su situación basal, consiguiéndose así el objetivo marcado como puntuación diana.

\section{CONCLUSIONES}

Aunque el ámbito clínico no parece, en principio, el más indicado para proporcionar educación para la salud, existen situaciones como la descrita ligada a la práctica enfermera, en la que resulta imprescindible para asegurar el autocuidado. Esto nos hace reflexionar sobre la importancia de identificar las necesidades formativas durante el ingreso hospitalario. A este respecto, consideramos 
cumplido nuestro objetivo dirigido a fomentar el autocuidado mediante actividades educativas para evitar infecciones tras el alta hospitalaria, al evaluar los conocimientos adquiridos por el paciente.

Finalmente, es evidente que el mejor tratamiento pierde efectividad si la persona no sabe o no esta motivada para responsabilizarse de sí, por lo que las sesiones educativas permiten al paciente situarse en un rol activo e involucrarse en su autocuidado disminuyendo la frecuencia de infecciones.

\section{RESPONSABILIDADES ÉTICAS}

Protección de personas y animales. Los autores declaran que en este estudio no se realizaron experimentos con personas o animales.

Confidencialidad. El participante firmó un consentimiento informado y se le ofreció la seguridad de resguardar sus datos personales.

Conflicto de intereses. Los autores declaran no tener conflicto de intereses.

Financiamiento. Ninguno.

\section{REFERENCIAS}

1. Esquinas C, Arance I, Pamplona J, Moraga A, Dorado JF, Angulo C. Tratamiento de la incontinencia urinaria de esfuerzo tras prostatectomía con el sistema masculino transobturador ajustable (ATOMS ${ }^{\circledR}$ ) con puerto escrotal premontado. Actas urol. esp. 2018; 42(7): 473-82.

https://dx.doi.org/10.1016/j.acuro.2018.02.005

2. Abrams P, Cardozo, Khoury S, Wein A. Incontinence. $4^{\text {th }}$ ed. Paris: Health Publication Ltd; 2009. https://bit.ly/3h3EIOL

3. Madrigal-Monge I, Girau-Alemany E. Actuación de enfermería en el tratamiento de la incontinencia urinaria tras prostatectomía radical mediante sling. Enfermería Integral. 2018; (119): 54-8. https://bit.ly/zasBeRm

4. Carvalho M, Machado FA, Andrade I. Terapias alternativas para la recuperación temprana de la continencia urinaria posterior a la prostatectomía: una revisión sistemática. Enfermería glob. 2018; 17(50): 542-56. https://bit.ly/3g9vChz

5. Rodríguez-Escobar F, Arañó-Bertrán P. Incontinencia urinaria post-prostatectomía: El esfínter artificial. Arch. Esp. Urol. 2009; 62(10): 838-44. https://bit.ly/3cLdUjt

6. Virasoro R, Capiel L, Ghisini D, González-Morales M, Cobreros Ch, Momesso A, et. al A. Esfínter urinario artificial transcorpóreo: ¿un recurso válido para el tratamiento de la incontinencia de orina post-prostatectomía radical?. Rev. Arg. de Urol. 2012; 77(3): 166-75. https://bit.ly/2KudpOt

7. Sandhu JS. Urinary Dysfunction in Prostate Cancer: A Management Guide. New York: Springer International; 2016.

8. Olaechea PM, Insausti J, Blanco A, Luque P. Epidemiología e impacto de las infecciones nosocomiales. Med Intensiva. 2010; 34(4): 256-67. https://doi.org/10.1016/j.medin.2009.11.013

9. National Clinical Guideline Centre (UK). Infection: Prevention and Control of Healthcare-Associated Infections in Primary and Community Care: Partial Update of NICE Clinical Guideline 2. NICE Clinical Guidelines, No. 139. London: Royal College of Physicians (UK); 2012. https://bit.ly/3eYpIjm

10. Arias-Arango AM, Valderrama-Maya MP, Parra-Pérez DM, Marín-Zuluaga JI, Mazo-Monsalve LM, Montoya-Zapata CP. Caracterización clínica y epidemiológica de los pacientes con infección del tracto urinario asociadas al cuidado de la salud. Invest Educ Enferm. 2012;30(1):28-34. https://bit.ly/33srdE6

11. Abasolo-Otegui I, Rezola-Aldaz B, Sarasola-González JC, Arrieta-Genua R, Gómez-Prieto Y, MúgicaEchevarría A, et al. Protocolo de sondaje vesical: Uso, inserción, mantenimiento y retirada. Enfuro. 2015; (128): 4-15. https://bit.ly/2xSTLZR 
12. Basak T, Uzun S. Comparison of 2 techniques used to obtain sterile urine specimens from urethral catheters. Clin Nurse Spec. 2013; 27(4): 194-7. https://doi.org/10.1097/nur.obo13e3182955756

13. Inman DM, Jacobson TM, Maxson PM, Wang H, Lohse CM. Effects of urinary catheter education for patients undergoing prostatectomy. Urol Nurs. 2013; 33(6): 289-98. https://wb.md/2UiulN6

14. Menéndez-González L, González-Sánchez GM. Cuidados del catéter urinario basados en la evidencia científica de Atención Primaria. RqR Enfermería Comunitaria. 2016; 4(1): 28-45. https://bit.ly/2XtesKX

15. Matarazzo JD, Weiss SM, Herd JA, Millar NE (Eds.), Behavioral health: A handbook of health enhancement and disease prevention. New York: Wiley; 1984.

16. Hernández-Díaz J, Paredes-Carbonel JJ, Marín-Torrens R. Cómo diseñar talleres para promover la salud en grupos comunitarios. Atención Primaria. 2014; 46 (1): 40-7.

https://doi.org/10.1016/j.aprim.2013.07.006

17. Pérez-Pérez L, Villa-Campos Z, Domínguez-Campos M. Educación enfermera al paciente portador de sonda vesical. Rev. Electrón. PortalesMédicos.com. 2017. https://bit.ly/3h1QiKd

18. Vicente-Herrero MT, Delgado-Bueno S, Bandrés-Moyá F, Ramírez-Iñiguez-de-la-Torre MV, CapdevillaGarcía L. Valoración del dolor. Revisión comparativa de escalas y cuestionarios. Rev. Soc. Esp. Dolor. 2018; 25(4): 228-36. http://dx.doi.org/10.20986/resed.2018.3632/2017

19. Prado-Solar LA, González-Reguera M, Paz-Gómez N, Romero-Borges K. La teoría déficit de autocuidado: Dorothea Orem punto de partida para calidad en la atención. Rev. Med. Electron. 2014; 36(6): 835-45 https://bit.ly/ziFUZcB

20. North American Nursing Diagnosis Association. Herdman T, Kamitsuru Sh. Diagnósticos enfermeros. Definiciones y clasificación 2015 - 2017. Madrid: Elsevier; 2017.

21. Butcher H, Bulechek G, McCloskey Dochterman J, Wagner C. Nursing Interventions Classification (NIC). $7^{\text {th }}$ ed. Philadelphia: Mosby; 2018.

22. Moorhead S, Johnson M, Maas ML, Swanson E. Nursing Outcomes Classification (NOC). $6^{\text {th }}$ ed. Philadelphia: Mosby; 2018.

23. Romanos-Calvo B, Casanova-Cartié N. La escala de Norton modificada por el INSALUD y sus diferencias en la práctica clínica. Gerokomos. 2017; 28(4): 194-9. https://bit.ly/3bW8mDB

24. González-Saura JL, Sandoval- Alcaraz MP, Ruiz-Vidal MJ, Carpintero-Moreno I, Hernández Agüera MC, Serna-Martínez AM. Recomendaciones al paciente con sondaje vesical. ICUE. 2016; 1(1). https://bit.ly/zayxEW3

25. Sánchez-Ormeño FJ. Triaje. Enfermería Ciudad Real. Ciudad Real España. Síndrome de reabsorción post-RTU prostática. 2016. https://bit.ly/2Vx1k1r

26. De Arco-Canoles O, Suarez-Calle Z. Rol de los profesionales de enfermería en el sistema de salud colombiano. Univ. Salud. 2018; 20(2): 171-82. https://doi.org/10.22267/rus.182002.121

27. Ángel-Jiménez G, López-Herrera FE, Restrepo-Múnera DM. La experiencia educativa del profesional de enfermería en el ámbito clínico. Investig. enferm. imagen desarro. 2013; 15(2): 9-29.

28. García-Moyano L. La ética del cuidado y su aplicación en la profesión enfermera. Acta Bioeth. 2015; 21 (2): 311-7. https://bit.ly/3gYsZR8

29. Marcos-Espino MP, Tizón-Bouza E. Aplicación del Modelo de Dorothea Orem ante un caso de una persona con dolor neoplásico. Gerokomos. 2013; 24(4): 168-77.

http://dx.doi.org/10.4321/S1134-928X2013000400005

30. Islas-Salinas P, Pérez-Piñón A, Hernández-Orozco G. Rol de enfermería en educación para la salud de los menonitas desde el interaccionismo simbólico. Enferm. univ. 2015; 12(1): 28-35.

https://doi.org/10.1016/j.reu.2015.05.005 